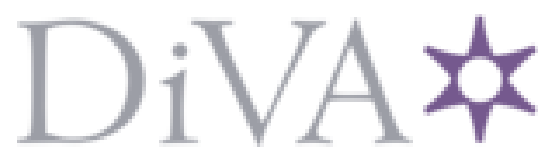

http://www.diva-portal.org

This is the published version of a paper presented at IEEE 15th International Conference on Environment and Electrical Engineering, June 10-13, Rome, Italy.

Citation for the original published paper:

Rebello, E., Almas, M., Vanfretti, L. (2015)

An Experimental Setup for Testing Synchrophasor-based Damping Control Systems.

In:

N.B. When citing this work, cite the original published paper.

Permanent link to this version:

http://urn.kb.se/resolve?urn=urn:nbn:se:kth:diva-170847 


\section{An Experimental Setup for Testing Synchrophasor-based Damping Control Systems}

\author{
Eldrich Rebello \\ KTH Royal Institute of Technology \\ Stockholm, Sweden \\ Email: rebello@kth.se
}

\author{
Muhammad Shoaib Almas \\ KTH Royal Institute of Technology \\ Stockholm, Sweden \\ Email: msalmas@kth.se
}

\author{
Luigi Vanfretti \\ KTH Royal Institute of Technology, Stockholm \\ Statnett SF, Oslo, Norway \\ Email: luigiv@kth.se, luigi.vanfretti@statnett.no
}

\begin{abstract}
Prototype Wide-Area Power system Oscillation Damping (WAPOD) controllers using synchrophasor data have been proposed, developed and tested in field demonstration projects. However, the development and testing process of these control systems has been confined to a few specific cases, the details of which have not been thoroughly reported in available literature. This paper details the development, construction and implementation of a real-time, Hardware-in-the-loop (HIL) test set-up for such a control system. A general purpose, phasorbased control algorithm is deployed on a Compact Reconfigurable Input/Output (cRIO) controller from National Instruments. The complete, closed-loop experimental set-up, the hardware used and the rationale behind different design choices are also documented. As this test-set-up uses IEEE C37.118 data over a TCP/IP network, the complete, real-time data path is examined. A realtime HIL test with the implemented controller is also presented and results are analysed.
\end{abstract}

\section{INTRODUCTION}

\section{A. Motivation}

Instances such as the Northeast blackout of August 2003 and the August 1996 Western North America (WECC) [1] blackout have been significantly disruptive events on large, interconnected power systems. The culprit behind the WECC blackout were low frequency, electromechanically induced, inter-area oscillations [1]. These oscillations involve the generators of one synchronous area oscillating against those of another area and are typically between $0.1-2 \mathrm{~Hz}$ in frequency. The fact that these modes are poorly damped [2] presents a danger to power systems with interconnections used for purposes such as power exchange.

\section{B. Previous Experiences}

The phenomenon of intra-area oscillations is well documented and has traditionally been solved using Power System Stabilizers (PSS). A PSS uses locally available signals and might not be very effective at damping inter-area modes with poor local observability [3] [4]. Wide-area control systems, as tested in demonstration projects in Norway [2] and China [5], have extended the control system of an existing device to receive and use synchrophasor (IEEE C37.118) data.

\section{Contributions}

The goal of this paper is to document the details of the design and construction of a real-time test set-up for a wide- area control system. The Phasor Power Oscillation (Phasor POD) damping algorithm [6] was implemented on a Compact Reconfigurable Input-Output controller (cRIO) [7] from National Instruments which was then tested with measurement input from a two-area model [8] executing in real-time. The entire physical test set-up is examined together with constraints of the present implementation. The results from one of the HIL experiments conducted are also presented. The design approach used is generic enough to serve as a starting point for other researchers to replicate in developing their own PMU-based controllers. The HIL test setup presented here is intended to serve as a test-bed that mimics real-world conditions as closely as possible.

Note:

The term 'real-time' as used in this work is identical to the sense used in the field of embedded control [9]. Though a simulated power network is used, it is executed in real-time so any external controller based on inputs from this system must behave in an identical manner when the same inputs are sourced from an actual power network. Both the real-time simulator and the cRIO controller clocks run simultaneously and as fast as an actual clock. While their internal clocks might differ in speed, they are synchronous and respect real-time constraints. The controller designed is thus able to provide feedback control to the power network model so as to affect the network at that point in time. Real-time is used in the sense that control output generated by the controller is guaranteed to be produced synchronously and in a fixed time frame which, in this case, is $50 \mu \mathrm{s}$. The timing limitations imposed are strict and if any delays occur, the controller is deemed to have failed.

\section{Paper Outline}

This paper is organised as follows. Section II outlines the damping algorithm selected, the controller architecture and introduces the power system model used. Section III presents details of the hardware used in the construction of the HIL test. Section IV presents the design choices behind the selection of the hardware used. A real-time HIL test and a sample result from it is presented in Section V. Conclusions are drawn in Section VI. This work does not cover details of the software written for the cRIO controllers or the SIMULINK models 
used. The focus, instead, is exclusively on the development and construction of the test setup used to verify the working of the developed controller. The reader is referred to [10] for details about the software and control architecture implemented in this work.

\section{BACKGROUND}

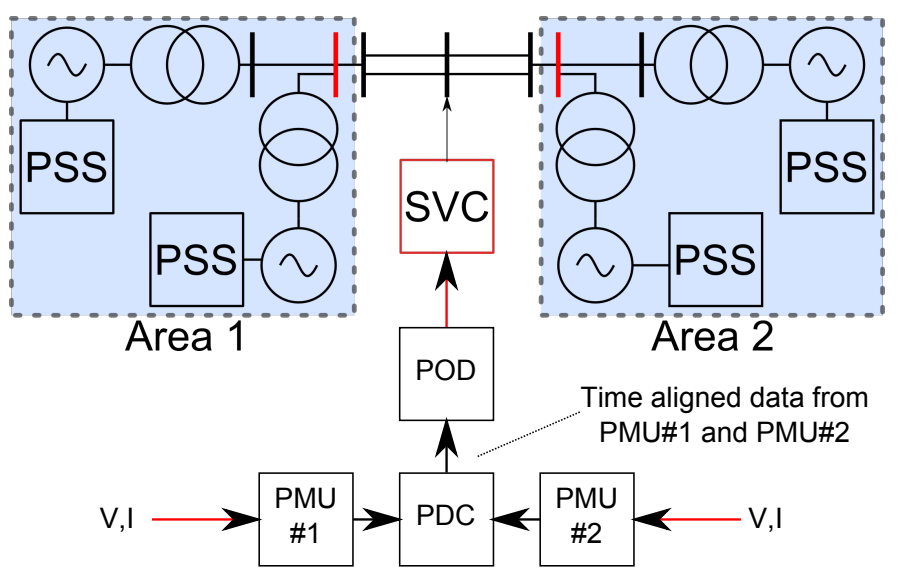

Fig. 1. Two-area model including an SVC, PMUs, PDC and the WAPOD Synchrophasor measurements taken from the red buses. Analogue signals are indicated with a red line.

\section{A. Two-Area Model \& Phasor POD}

The Phasor-POD algorithm essentially separates an input signal into average-valued and oscillating components [6]. The oscillating component, when suitably phase-shifted, can act as a damping input to a controllable device. This algorithm [6] was selected due to its wide applicability and because it does not depend on the power network's topology which can change during operation. Typical model-linearisation-based damping algorithms rely on computer-intensive calculations, often valid for a limited range of operating points, and are hence not suitable for real-time implementation. In contrast, the Phasor-POD algorithm only requires knowledge of the inter-area oscillation frequency, which is generally known from system studies or can be determined from synchrophasor measurements [11]. This algorithm has been demonstrated to work with a two-area model (see Fig. 1) where it acts as a modulating input to a flexible AC transmission system (FACTS) device [12]. The SIMULINK implementation of the Phasor-POD algorithm as presented in [12] was re-written in LabView and deployed on an NI-cRIO9081. The reader is referred to [8] for details of the original two-area model used, which has been modified for this work.

\section{B. Controller Architecture}

Figure 2 details the three-layer controller architecture as implemented. The two-area model executes in real-time on the OPAL-RT simulator. Phasor Measurement Units (PMUs) are connected to the analogue outputs of the real-time simulator. The synchrophasor data stream that these PMUs generate is parsed on a desktop computer (Labelled 1 in Fig. 2). This data is then sent over a TCP/IP network to the cRIO running the control algorithm. The Phasor-POD algorithm is deployed on the cRIO's FPGA which generates an analogue damping signal. This signal is fed back to the OPAL-RT simulator thus completing the HIL loop.

\section{SmarTS Lab}

All the hardware and software detailed in this work is available in the SmarTS Lab at KTH, Stockholm. For full details of the lab, all its equipment and capabilities, the reader is referred to [13]. This work presents relevant components that form part of the HIL experiment described here and only touches upon their capabilities. A labelled but simplified view of the SmarTS Lab is shown in Fig. 6.

\section{HARDWARE \& TEST SETUP}

Figure 4 presents the data path and a simplified outline of the entire real-time HIL test conducted. This was the final test setup at which the authors arrived at considering limitations of the hardware and software available. These are covered in the next section.

\section{A. OPAL-RT Simulator}

The eMEGASIM real-time simulator from OPAL-RT [14] was at the core of the HIL test. The simulator executed the two-area SIMULINK model in real-time and facilitated interfacing hardware with the model through its analogue input and output terminals. Details of these terminals are as follows:

- Analogue Outputs: $32(+/-16 \mathrm{~V}$ and $+/-10 \mathrm{~mA})$

- Analogue Inputs: 128 (+/-100 V and +/-10 mA)

The simulator computed and updated values for all variables in the two-area model every $50 \mu \mathrm{s}$. This time step was selected to allow for the assumption of linearity of power system variables between each integration time step. Updated values were written to the analogue outputs and input values read at the analogue inputs every $50 \mu \mathrm{s}$. The SIMULINK model was developed and edited on a workstation computer and the same computer was used to monitor output from the simulator while the model was running.

\section{B. cRIO Real-Time Controllers}

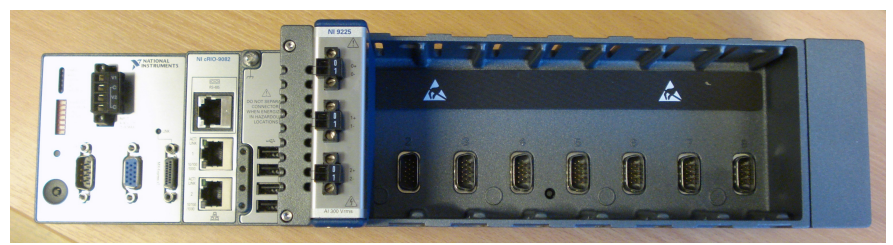

Fig. 3. cRIO9081 showing an NI9225, three-channel, analogue voltage input module and seven empty add-on module slots

Two different cRIO models were used in this particular HIL experiment. One model, the NI-cRIO9081, was used to run the Phasor-POD algorithm in real-time. Two NI-cRIO9076s were deployed as PMUs. Figure 3 shows the NI-cRIO9081 with an analogue voltage input add-on module connected. The data 


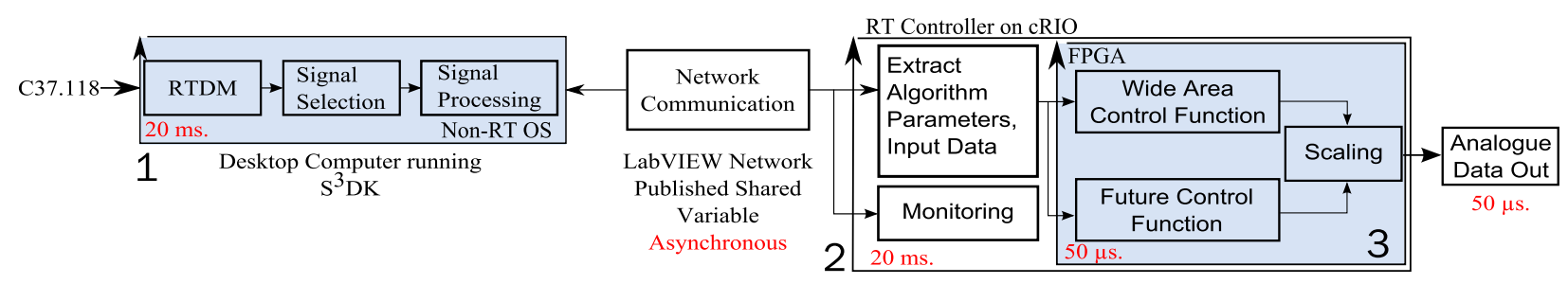

Fig. 2. Three layer controller architecture as implemented. Layer 1 is the desktop computer used to parse the C37.118 data stream. Layers 2 and 3 run on the real-time and FPGA sections of the cRIO. Loop rates are indicated in red.

generated by the Phasor-POD algorithm was sent back to the real-time simulator as an analogue signal. Alternate control hardware can be expected to perform equally well as long as the timing requirements are rigidly enforced.

PMUs: Two NI-cRIO9076s were used as PMUs, each with a four-channel, analogue voltage input module and a threechannel, analogue current input module. The inputs to the PMUs were three-phase currents and voltages and a GPS signal for time-synchronisation. PMU software from National Instruments was run on both. Each PMU generated an IEEE C37.118-compliant synchrophasor data stream which was sent over a TCP/IP network. The reporting rate of the PMUs was 50 messages per second hence new measurements were available to the controller every $20 \mathrm{~ms}$.

Using analogue signals and hardware PMUs allows for closely mimicking a real-world deployment scenario. The module ratings were $0-300 \mathrm{~V}$ and $0-5 \mathrm{~A}$ with 24 bit resolution each [7] for the NI9225 and NI9227 respectively. To reduce subsequent computation burden, the PMUs were also configured to compute and report values of active power. The synchrophasor stream from each PMU was sent to a Phasor Data Concentrator (PDC) [15] where the streams were time aligned for subsequent use.

Real-Time Phasor-POD Algorithm: The FPGA of the NIcRIO9081 was used to run the Phasor-POD algorithm in real-time. Though the NI-cRIO9081 has a real-time microprocessor in addition to the FPGA, the latter was chosen to run the Phasor-POD algorithm. This was because the FPGA runs at $400 \mathrm{MHz}$ and is thus capable of a deterministic response time in the order of nanoseconds. The loop rate chosen was $50 \mu$ s so that the data output rate of the FPGA was identical to the read rate on the real-time simulator's input.

\section{Analogue Signal Amplifiers}

The SMRT1 Single Phase Relay Tester [16] was used as an analogue signal amplifier. Each individual amplifier had a single current and single voltage input. To drive the inputs of two PMUs, six amplifier units were required. The inputs to the amplifiers were the low-level analogue signals extracted from the real-time simulator. The outputs of the amplifiers were wired to the analogue input modules of the PMUs. Table I lists the ratios used. To avoid saturation in the amplifiers, the outputs from the real-time simulator were limited to $\pm 10 \mathrm{~V}$.
TABLE I

AMPLIFIER INPUTS AND OUTPUTS

\begin{tabular}{|c|c|c|}
\hline & Input, from simulator & Amplified Output \\
\hline Voltage & $\pm 10 \mathrm{~V}$ & $\pm 100 \mathrm{~V}$ \\
\hline Current & $\pm 20 \mathrm{~mA}$ & $\pm 1 \mathrm{~A}$ \\
\hline
\end{tabular}

\section{Phasor Data Concentrator}

The Phasor Data Concentrator (PDC) consisted of a network connected desktop computer running PDC software from Schweitzer Engineering Laboratories (SEL) [15]. The PDC allowed for data from multiple synchrophasor streams to be concentrated, time-aligned and used. It also had data logging functionality which was used to analyse the overall control system performance.

\section{Hardware Design Choices}

Figure 4 shows the final HIL test setup which was used to evaluate the performance of the real-time controller. This design was arrived at after numerous changes to the setup. This section outlines the most significant changes made together with the rationale behind them.

\section{A. Analogue Signal Amplifiers}

The initial setup envisaged connecting the analogue outputs of the real-time simulator directly to the inputs of the PMUs. This approach simplified wiring but had to be abandoned due to a poor signal-to-noise ratio. The main reason for this poor signal-to-noise ratio was the fact that a very small portion of the dynamic range of the PMU's input modules was being used. A $0-10 \mathrm{~V}$ signal was being read by a voltage module rated for $0-300 \mathrm{~V}$ and a $0-20 \mathrm{~mA}$ signal was being read by a module rated for $0-5 \mathrm{~A}$. This resulted in the POD algorithm producing a large error signal even at steady state.

Using an amplified signal as a PMU input greatly improved the signal-to-noise ratio and thus the performance of the Phasor-POD algorithm. Though the amplifiers improved the signal-to-noise ratio, additional scaling factors were introduced in the simulation and in the PMUs. For instance, the analogue output of the real-time simulator had to be limited in magnitude to avoid problems such as amplifier saturation. Also, CT and PT ratios in the PMUs were used to account for scaling factors in other sections of the HIL test. Simply scaling up any signal also increases the absolute magnitude of contained 


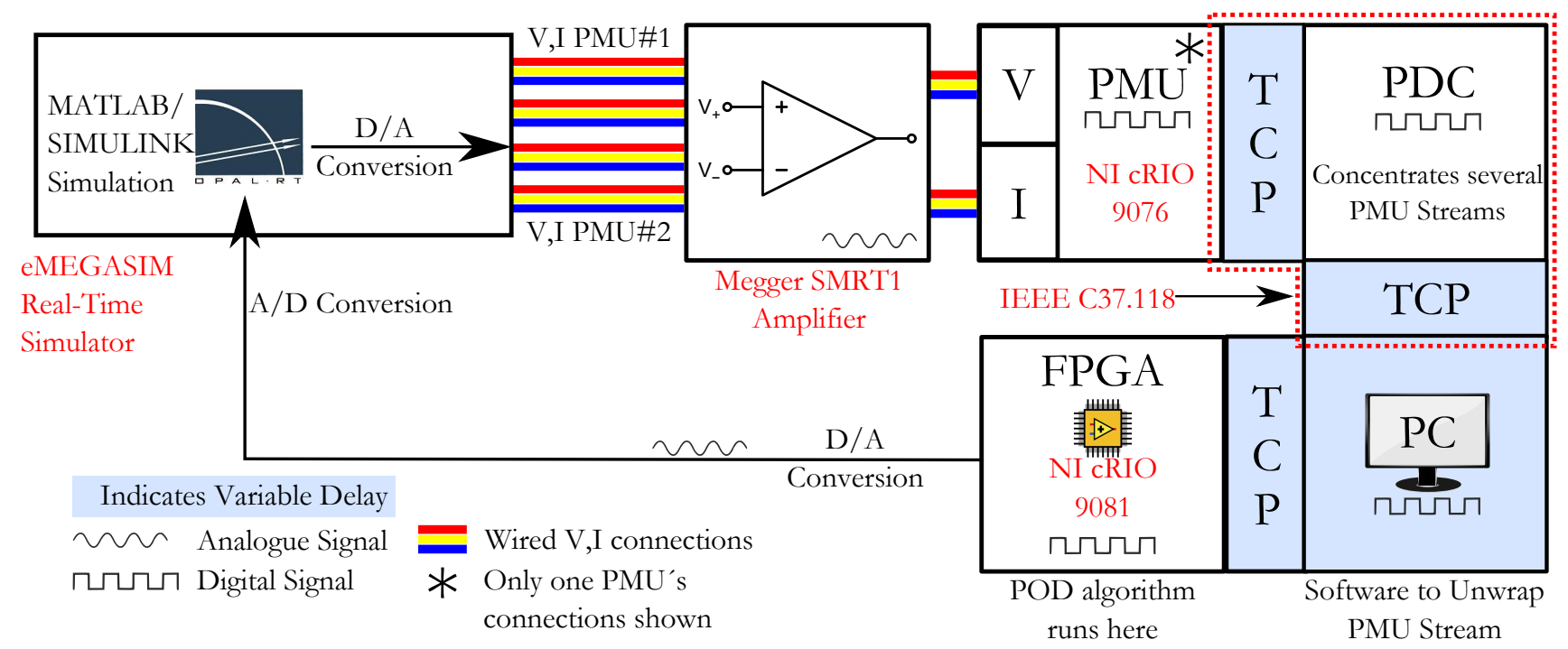

Fig. 4. Data path and experiment out ling of HIL test. Hardware used is indicated in red text. Note that two PMUs are used though one is indicated. The section where synchrophasor data is used is indicated with a dotted line.

noise. This is not expected to be a problem when using actual CTs and PTs.

\section{B. FPGA}

The Phasor-POD algorithm was implemented on the FPGA of the NI-cRIO9081. This was done despite the challenges of writing FPGA code and despite the fact that the algorithm could have been executed on the real-time section of the controller. Two reasons were behind this choice. One was that the real-time section of the cRIO9081 handles asynchronous TCP/IP communication. This had to be incorporated in to a deterministic control loop, which would also run the control algorithm. Any delays in the communication network may result in delayed control output. The second problem was that the fastest loop rate that the real-time controller was capable of was $1 \mathrm{~ms}$. This was achievable only under ideal conditions with low processor load and minimum network delay. The real-time simulator expected input from the controller every $50 \mu \mathrm{s}$, a rate that the real-time controller could not match. By leaving the task of network communication to the real-time controller, the FPGA was used to run the control algorithm together with a sample-and-hold algorithm and produce output every $50 \mu \mathrm{s}$.

\section{Desktop Computer}

An ideal controller would be able to parse the synchrophasor stream, extract measurement data and perform control action autonomously. However, no software was available that could run on the cRIO and parse the synchrophasor stream. Software [17] was, however, available and could perform this function on a desktop computer. The solution implemented here thus has a desktop computer running a multi-tasking operating system to extract data from the synchrophasor stream. This data is then sent to the cRIO over the TCP network. In the scenario where data from multiple PMUs is used, computations such as the voltage phase angle difference are performed on this computer.

\section{HARDWARE-IN-THE-LOOP TEST}

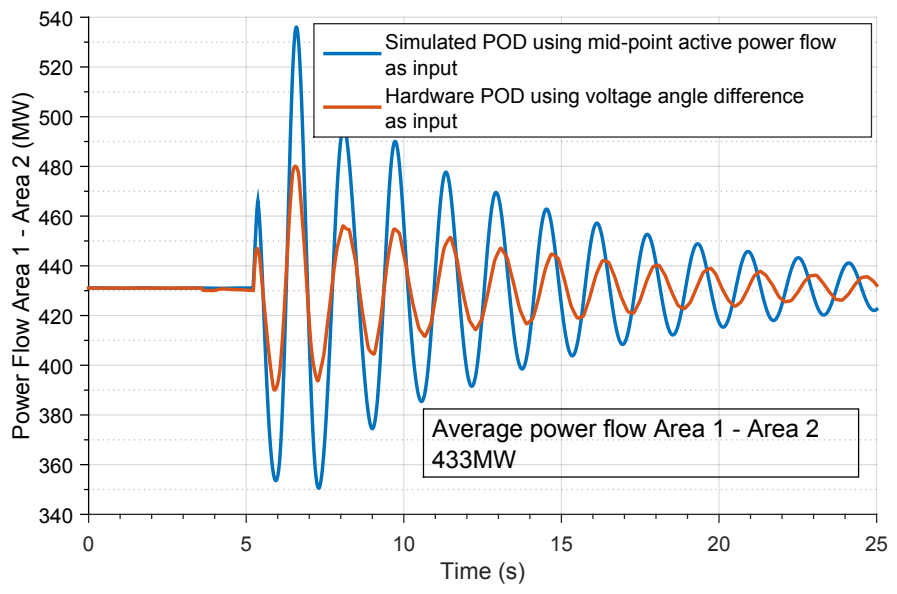

Fig. 5. Damping action in inter-area power flow. Blue plot shows maximum damping action achievable with active power input. Note that improved damping performance is achieved with the HIL controller and wide-area data such as the voltage angle difference.

A setup identical to that outlined in Figure 4 was constructed and used to verify the working of the hardware controller. As opposed to a real-time simulation, a real-time HIL test has sources of delay and noise. Sources of stochastic delay are indicated in blue in Fig. 4. Three-phase waveforms are extracted from the edges of Area 1 and Area 2 as indicated in Fig. 1 and are sent to two PMUs via analogue amplifiers. The PMUs generate synchrophasor data streams which are sent to a PDC over a TCP/IP network. The PDC time-aligns both streams and retransmits them over the same TCP/IP network. The streams are received on a desktop computer 
running LabView. Here, measurement data is extracted from the streams and is sent to the control algorithm running on the cRIO. The cRIO receives this data and passes it to the built-in FPGA which runs the Phasor-POD algorithm. The FPGA uses an analogue voltage output module to generate a control signal which is wired back to the real-time simulator. After scaling, this signal is reintroduced in the SImUlinK model running in real-time.

The setup described above is modular enough to allow entire sections to be replaced with their equivalents or removed altogether. The real-time simulator, for example, can be replaced with an actual power system and a controllable device such as an SVC. Even the choice of controllable device is not limited and the control signal generated by the real-time controller can just as well be used to modulate a generator's automatic voltage regulator (AVR) input. In the case where an actual power system is used, the PMU current and voltage inputs should be from CTs and VTs respectively. In the event that only one PMU is used, the PDC becomes redundant and can be removed.

Results from the real-time HIL test are presented in Figure 5. The Phasor-POD algorithm running on the cRIO can use a variety of signals extracted (or calculated) from the synchrophasor stream to drive the controller. The inter-area mode is excited at approximately $\mathrm{t}=5 \mathrm{~s}$ by momentarily changing the voltage reference of one generator in Area 1 before returning it to normal. In both plots in Fig. 5, the SVC provides the only stabilising action to an otherwise unstable [8] system. The blue plot shows the maximum damping achievable when using active power flow as the input to the POD algorithm. The orange plot shows the damping performance achieved when using the voltage angle difference as a damping input. As evident in Fig. 5, the Phasor-POD algorithm on the cRIO is able to restore the system to stability in both cases presented despite the errors introduced by signal noise, measurement error and network transport delay. This presents a strong case for using wide-area signals such as the voltage angle difference between the two areas are essential to achieve improved damping performance.

The advantage of such a controller is the ability to use wide-area signals as damping inputs thus improving inter-area mode observability and enhancing damping performance. Figure 5 is not meant to serve as a comparison but rather, a confirmation that real-time damping can be achieved using commercially available, general purpose controllers.

The experimental setup detailed here was designed for testing one particular algorithm. It is, however, significantly generic and modular that portions of it can be replaced or removed entirely as described previously. The controller used here was implemented using proprietary hardware and software from National Instruments. The support, documentation and software from National Instruments greatly accelerated the development and testing of this prototype. The authors, however, would like to develop similar controllers on open hardware platforms such as the Raspberry Pi or Arduino.

\section{CONCLUSION}

An outline of a generic, real-time, HIL test setup used to test synchrophasor data-driven control systems was presented. The hardware used in the construction of this test together with the design decisions that influenced these choices are presented. The results from one HIL experiment were described and serve to verify the working of the real-time, hardware implementation of the Phasor-POD algorithm. The authors hope that the contents of this article will help other researchers develop their own experimental setups by documenting the difficulties and solutions to practical problems and limitations faced by the authors.

\section{ACKNOWLEDGEMENT}

The work of E. Rebello \& M.S. Almas was supported by the STRON $g^{2}$ rid project, funded by Nordic Energy Research. L. Vanfretti is supported by the $\mathrm{STRON} g^{2}$ rid project, funded by Nordic Energy Research and by Statnett SF, the Norwegian transmission system operator.

\section{REFERENCES}

[1] North American Electric Reliability Council "Review of selected 1996 Electric System Disturbances in North America" August 2002. Available Online: http://www.nerc.com/pa/rrm/ea/System $\backslash \% 20$ Disturbance $\backslash$ \%20Reports20DL/1996SystemDisturbance.pdf

[2] K. Uhlen, L. Vanfretti, M.M. De Oliveira, A.B. Leirbukt, V. H. Aarstrand and J. O. Gjerde "Wide-Area Power Oscillation Damper implementation and testing in the Norwegian transmission network" in Power and Energy Society General Meeting, 2012 IEEE pp. 1-7

[3] L. Vanfretti, Y. Chompoobutrgool, and J.H. Chow, "Chapter 10: Inter-Area mode analysis for large power systems using synchrophasor data" Book Chapter, in Coherency and Model Reduction of Large Power Systems, Joe H. Chow (Ed.), Springer, 2013.

[4] M. E. Aboul-Ela, A. A. Sallam, J. D. McCalley and A. A. Fouad, "Damping controller design for power system oscillations using global signals" IEEE trans. on Power Systems, Vol. 11, No. 2, May 1996, pp. 767-773

[5] Li Peng and Wu Xiaochen and Lu Chao and Shi Jinghai and Hu Jiong and He Jingbo and Zhao Yong and Aidong Xu "Implementation of CSG's wide-area damping control system: Overview and experience" in Power Systems Conference and Exposition, 2009. PSCE '09. IEEE/PES pp. 1-9

[6] L. Ängquist and C. Gama "Damping algorithm based on phasor estimation" in Power Engineering Society Winter Meeting, 2001. IEEE, Volume 3, pp. 1160 - 1165

[7] Operating Instructions and Specifications Compact RIO NI cRIO9075/9076 \& NI cRIO-9081/9082 National Instruments, Available Online at http://www.ni.com/

[8] M. Klein, J. G. Rogers and P. Kundur "A fundamental study of inter-area oscillations in Power Systems" IEEE Trans, PWRS, no. 6, pp. 914-921, 1991.

[9] Ben-Ari, M., "Principles of concurrent and distributed programming", Prentice Hall, 1990. ISBN 0-13-711821-X. Ch16, pp. 164

[10] E. Rebello, L. Vanfretti and M.S Almas "PMU-based real-time damping control system software and hardware architecture synthesis and evaluation" IEEE PES GM, Denver, Colorado, July 2015, in press

[11] M.Crow, M. Gibbard, A. Messina, J. Pierre, J. Sanchez-Gasca, D. Trudnowski, D. Vowles "Identification of electromechanical modes in power systems" IEEE Task Force Report, Special Publication TP462, June2012

[12] M. Shoaib Almas and L. Vanfretti, "Implementation of conventional PSS and phasor-based POD for power stabilizing controls for real-time simulation” IEEE IES IECON14, 29 Oct-1 Nov, 2014, Dallas, USA. 
[13] M.S. Almas, M. Baudette, L. Vanfretti, S. Lovlund and J.O. Gjerde "Synchrophasor network, laboratory and software applications developed in the STRON $g^{2}$ rid project" PES General Meeting, Conference Exposition, July 2014 IEEE pp 1 - 5

[14] eMEGAsim PowerGrid Real-Time Digital Hardware in the Loop Simulator Opal RT Available Online: http://www.opal-rt.com/

[15] SEL SynchroWAVE Phasor Data Concentrator (PDC) Software Available Online at https://www.selinc.com/ Copyright 2015 Schweitzer Engineering Laboratories, USA
[16] SMRT1 Single Phase Relay Test System Data Sheet, Available Online http://www.megger.com/common/documents/SMRT1_DS_ en_V10.pdf Retreived 22.12.2014, 2014 () Megger

[17] L. Vanfretti, V.H. Aarstrand, M. Shoaib Almas, V. S. Perić and J.O Gjerde "A Software development toolkit for real-time synchrophasor applications" IEEE PES Grenoble PowerTech, 2013

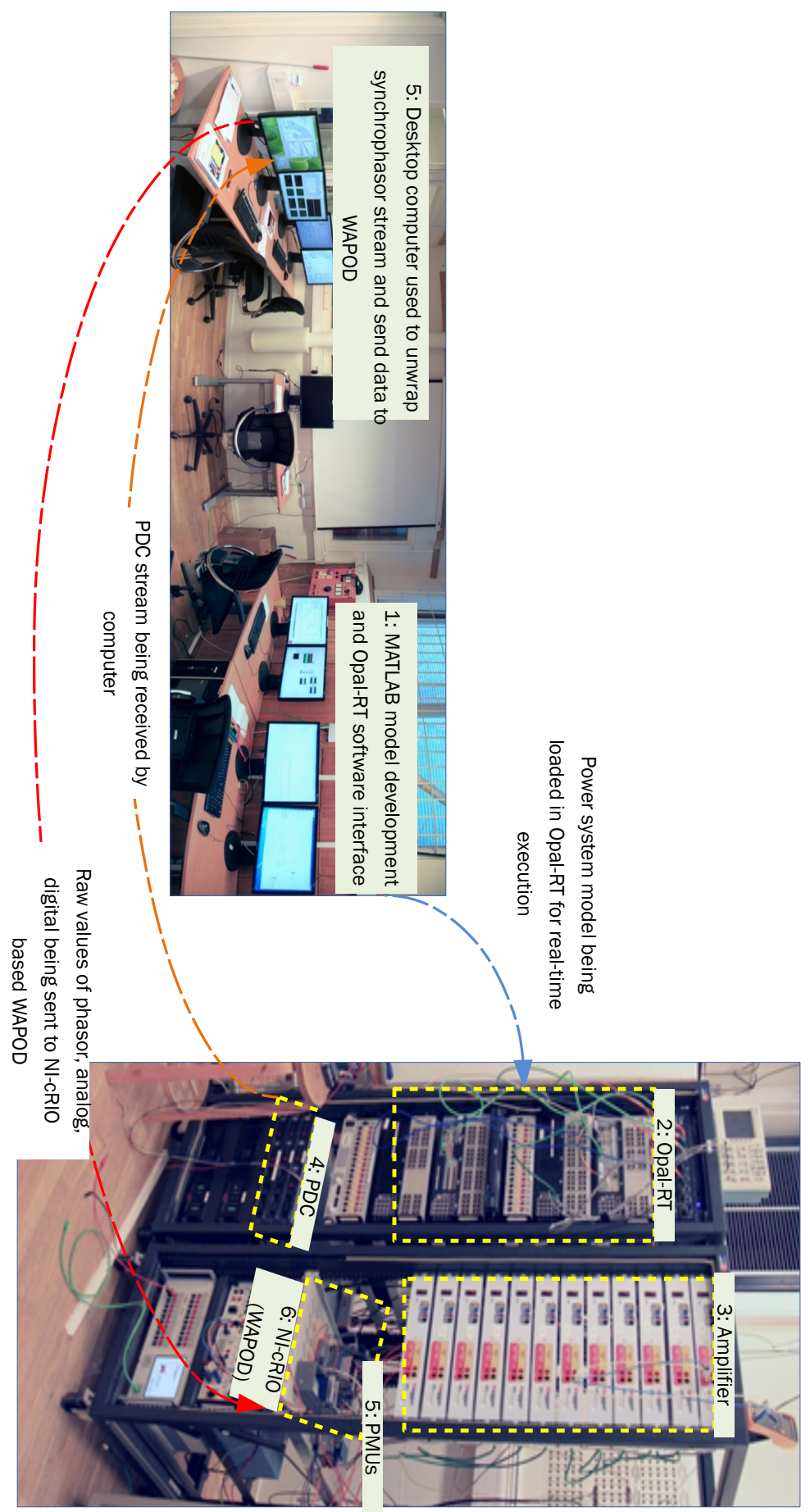

Fig. 6. Outline of the SmarTS Lab. Visible on the right is the OPAL-RT real-time simulator, the cRIO tray, the PDC, PMUs and the amplifiers. On the left are the development computers. 